\title{
Altered Inhibition-Related Frontolimbic Connectivity in Obsessive-Compulsive Disorder
}

\author{
Laura S. van Velzen, ${ }^{1,2}$ Stella J. de Wit, ${ }^{1,2,3}$ Branislava Ćurĉić-Blake, ${ }^{4}$ \\ Daniëlle C. Cath, ${ }^{5,6}$ Froukje E. de Vries, ${ }^{1,2,3}$ Dick J. Veltman, ${ }^{1,2}$ \\ Ysbrand D. van der Werf, ${ }^{2,3,7}$ and Odile A. van den Heuvel ${ }^{1,2,3 *}$ \\ ${ }^{1}$ Department of Psychiatry, VU University Medical Center, Amsterdam, the Netherlands \\ ${ }^{2}$ Neuroscience Campus Amsterdam (NCA), Amsterdam, the Netherlands \\ ${ }^{3}$ Department of Anatomy and Neurosciences, VU University Medical Center, \\ Amsterdam, the Netherlands \\ ${ }^{4}$ Department of Neuroscience, Neuroimaging Center, University Medical Centre Groningen, \\ Groningen, the Netherlands \\ ${ }^{5}$ Altrecht Academic Anxiety Center, Utrecht, the Netherlands \\ ${ }^{6}$ Division of Clinical and Health Psychology, Utrecht University, Utrecht, the Netherlands \\ ${ }^{7}$ Netherlands Institute for Neuroscience, Netherlands Academy of Sciences,
} Amsterdam, the Netherlands

\begin{abstract}
Background: Recent studies have shown that response inhibition is impaired in patients with obsessive-compulsive disorder and their unaffected siblings, suggesting that these deficits may be considered a cognitive endophenotype of obsessive-compulsive disorder. Structural and functional neural correlates of altered response inhibition have been identified in patients and siblings. This study aims to examine the functional integrity of the response inhibition network in patients with obsessive-compulsive disorder and their unaffected siblings. Methods: Forty-one unmedicated patients with obsessive-compulsive disorder, 17 of their unaffected siblings and 37 healthy controls performed a stop signal task during functional magnetic resonance imaging. Psycho-physiological interaction analysis was used to examine functional connectivity between the following regions of interest: the bilateral inferior frontal gyri, presupplementary motor area, subthalamic nuclei, inferior parietal lobes, anterior cingulate cortex, and amygdala. We then used dynamic causal modeling to investigate the directionality of the networks involved. Results: Patients, and to a lesser extent also their unaffected siblings, show
\end{abstract}

Additional Supporting Information may be found in the online version of this article.

Contract grant sponsor: NARSAD young investigator award of the Brain and Behavior Research Foundation (to O.A.v.d.H.); Contract grant sponsor: Netherlands Organization for Scientific Research; Contract grant sponsor: NWO-ZonMw VENI; Contract grant number: 916.86 .036 (to O.A.v.d.H.); Contract grant sponsor: NWO-ZonMW AGIKO stipend; Contract grant number: 920-03542 (to F.E.d.V.); Contract grant sponsor: Netherlands Brain Foundation; Contract grant number: (2010(1)-50); Contract grant sponsor: Stichting tot Steun VCVGT; Contract grant number: STO957

*Correspondence to: O.A. van den Heuvel, MD PhD; Department of Psychiatry, VU University Medical Center, PO Box 7057, 1007 MB, Amsterdam, The Netherlands. Email: oa.vandenheuvel@vumc.nl

Conflict of interest: None.
Ethical Standards: The authors assert that all procedures contributing to this work comply with the ethical standards of the relevant national and institutional committees on human experimentation and with the Helsinki Declaration of 1975, as revised in 2008.

Copyright transfer: This work has neither been published previously, nor is under simultaneous review by a second journal. Data used in this paper, however, has previously been used for different analyses and results of these analyses have been published in the American Journal of Psychiatry, 169 (10) p.11001108.

DOI: $10.1002 / \mathrm{hbm} .22898$

Published online 15 July 2015 in Wiley Online Library (wileyonlinelibrary.com). 
altered connectivity between the inferior frontal gyrus and the amygdala during response inhibition. The follow-up dynamic causal modeling suggests a bottom-up influence of the amygdala on the inferior frontal gyrus in healthy controls, whereas processing occurs top-down in patients with obsessive-compulsive, and in both directions in siblings. Conclusions: Our findings suggest that amygdala activation in obsessivecompulsive disorder interferes differently with the task-related recruitment of the inhibition network, underscoring the role of limbic disturbances in cognitive dysfunctions in obsessive-compulsive disorder. Hum Brain Mapp 36:4064-4075, 2015. @ 2015 Wiley Periodicals, Inc.

Key words: effective connectivity; obsessive-compulsive disorder; response inhibition; dynamic causal modeling; psycho-physiological interaction

\section{INTRODUCTION}

Obsessive-compulsive disorder is a neuropsychiatric disorder characterized by distress and anxiety provoking obsessions (repetitive intrusive thoughts) and compulsions (repetitive ritualistic behaviors) that are performed to diminish anxiety. This disorder is thought to affect between two and three percent of the population [Fontenelle et al., 2006; Ruscio et al., 2010]. Current neurobiological models implicate dysfunction of frontal-striatal, frontoparietal and frontolimbic circuits in the etiology of this disorder [Milad and Rauch, 2012; van den Heuvel et al., in press]. The models propose an imbalance between interacting affective, cognitive, and sensorimotor corticostriato-thalamo-cortical (CSTC) circuits, and altered connectivity between these circuits and the amygdala [Milad and Rauch, 2012; van den Heuvel et al., in press]. The specific pathways by which (para)limbic regions including the amygdala may influence cognitive functioning have yet to be identified.

One task that activates multiple areas in the cognitive CSTC circuit is the stop signal task [Logan and Cowan, 1984]. This task probes response inhibition, the ability to inhibit a motor response when it is no longer required or inappropriate. Impaired response inhibition may underlie repetitive behaviors, such as the compulsions seen in obsessive-compulsive disorder. Studies have shown response inhibition deficits in patients with this disorder, as indicated by increased commission errors during go-no go task performance [Bannon et al., 2002] and increased stop signal reaction times (SSRT) in the stop signal task [Chamberlain et al., 2006]. Deficits in response inhibition

\section{Abbreviations}

BA Brodmann area

BMA Bayesian model averaging

BMS Bayesian model selection

DCM Dynamic causal modeling

IFG Inferior frontal gyrus

PPI Psycho-physiological interaction

Pre-SMA Presupplementary motor area

SSRT Stop signal reaction time have also been observed in unaffected siblings of patients with obsessive-compulsive disorder, suggesting that the response inhibition impairment is state-independent and strengthening the notion of impaired response inhibition as a neurocognitive endophenotype of obsessive-compulsive disorder [Chamberlain et al., 2007].

Neuroimaging studies have shown that performance of the stop signal task is dependent on indirect and hyperdirect CSTC pathways in healthy subjects [Aron, 2011; Aron and Poldrack, 2006; Chambers et al., 2009]. The inferior frontal gyrus (IFG) and presupplementary motor area (pre-SMA) are crucial for response inhibition [Chambers et al., 2006; Chen et al., 2009] and appear to initiate the neural stop signal [Aron, 2011]. In the indirect pathway, the signal that is initiated in the frontal regions, reaches the thalamus via the striatum, globus pallidum and subthalamic nucleus, while it reaches the thalamus through the subthalamic nucleus directly in the hyperdirect pathway. Thalamic output then has an inhibitory effect on the primary motor cortex and suppresses the initiated motor response. Thus, the indirect and hyperdirect pathways both mediate response inhibition through their inhibitory effect on the motor cortex.

Several studies in patients with obsessive-compulsive disorder have observed structural deficits in frontostriatal regions associated with response inhibition, including the IFG [De Wit et al., 2014]. We have also previously reported decreased activation of the right inferior parietal and right IFG in OCD patients during performance of a response inhibition task [de Wit et al., 2012]. We additionally found increased task-related activation of the left pre-SMA in OCD patients and their siblings, indicating that compensatory pre-SMA hyperactivity during response inhibition may be an endophenotype of obsessive-compulsive disorder. Other functional imaging studies in obsessive-compulsive disorder have also reported decreased inhibition-related activation of the IFG [Page et al., 2009; Woolley et al., 2008], striatum [Kang et al., 2013; Page et al., 2009; Woolley et al., 2008], and thalamus [Page et al., 2009; Woolley et al., 2008], although one study reported increased inhibition-related activation of these areas in patients with obsessive-compulsive disorder [Maltby et al., 2005]. 
To date, no studies have focused on the functional connectivity patterns during response inhibition in patients with obsessive-compulsive disorder. Recently, Kang et al. [2013] examined how abnormal resting state connectivity was related to inhibitory performance on the stop signal task. This group found greater connectivity between the right middle cingulate cortex and right parahippocampal cortex, and between the right middle cingulate cortex and left caudate at rest in patients with obsessive-compulsive disorder. Connectivity between the middle cingulate cortex and the parahippocampal cortex correlated negatively with SSRT. Another study examined connectivity during performance of a related inhibition task, the Stroop task, using dynamic causal modeling and found increased inhibitionrelated connectivity between the anterior cingulate cortex and the dorsolateral prefrontal cortex in patients with obsessive-compulsive disorder [Schlösser et al., 2010].

To gain insight in the mechanisms underlying the response inhibition impairment in patients with obsessivecompulsive disorder and their unaffected siblings and to further elucidate the results from our previous fMRI study [de Wit et al., 2012], we aimed to examine functional connectivity patterns during the performance of the stop signal task in patients with obsessive-compulsive disorder, their unaffected siblings and healthy controls. We hypothesized that impaired response inhibition in patients with obsessive-compulsive disorder and their unaffected siblings is related to altered connectivity between the IFG and preSMA, as these regions are critical for response inhibition performance and show abnormal activity in patients and siblings during task performance. Based on previous studies [de Vries et al., 2014; van den Heuvel et al., 2011] we also hypothesized altered connectivity between the amygdala and prefrontal cortex during task performance in OCD patients and their unaffected siblings.

\section{MATERIALS AND METHODS}

\section{Participants}

Participants in this study were 41 medication-free patients with obsessive-compulsive disorder, 17 of their unaffected siblings, and 37 healthy controls. Inclusion and exclusion criteria have been reported elsewhere [de Wit et al., 2012]. To briefly summarize, participants were matched for age (patients: mean 38.6 y (standard deviation 9.8); siblings: mean 38.3 y (13.4); controls: mean 39.7 y (11.6); $(F[2,92]=0.01, P=0.871)$, gender (patients: $21 / 41$ males; siblings: 12/17 males; controls: 18/37 males); $\left(\chi^{2}=2.4, P=0.297\right)$, and educational level [patients: mean 5.9 y (1.9); siblings: mean 5.7 y (1.3); controls: mean 5.9 y (1.9) $(F[2,92]=0.2, P=0.896)]$ (see de Wit et al., 2012, Table I). Patients with obsessive-compulsive disorder scored higher on clinical measures of depression than controls (Montgomery-Åsberg Depression Rating Scale scores: patients: mean 11.5 (8.1); siblings: mean 1.9 (3.5); controls: mean $0.8(1.0) \quad(F[2,92]=0.26, \quad P<0.001) . \quad$ As expected, patients with obsessive-compulsive disorder showed higher disease severity scores (Yale-Brown Obsessive Compulsive Scale scores: patients: mean 21.9 (6.1); siblings: mean 0.1 (0.2); controls: mean $0.0(0.0) ;(F[2,92]=83.8 . P<$ $0.001)$, and reported more obsessive-compulsive disorder symptoms than siblings and controls (obsessive-compulsive inventory revised total score: patients: mean 24.4 (12.0); siblings: mean 4.1 (1.1); controls: mean 3.2 (4.8); $(F[2,92]=62.4, P<0.001)$. More than half of the patients also met criteria for one or more other axis-I disorders $(54 \%)$, but the presence of comorbid disorders was not associated with task performance or obsessive-compulsive disorder severity. All subjects provided written informed consent and all procedures were approved by the medical ethical review board of the VU University Medical Center (Amsterdam, the Netherlands).

\section{Stop Signal Task}

Participants performed a stop signal task, which is described in detail elsewhere [Logan, 1994], during functional magnetic resonance imaging. To summarize, during this task subjects were asked to press their right or left index finger on a button to indicate the direction of an arrow (go signal), as fast and accurate as possible. On 20 percent of trials a stop signal appeared (stop trials) after the go signal and subjects were instructed to inhibit the initiated response. The delay between the go signal and the stop signal varied in such a way that the response was successfully stopped in about 50 percent of the stop trials. It was then possible to calculate the stop signal reaction time, the duration of the stop process, for each participant.

\section{Analysis of Task Performance}

Behavioral data analysis for post hoc analyses was performed in SPSS version 20 (IBM), see for main groupeffects our previous report [de Wit et al., 2012]. The statistical threshold was set at $P<0.05$. Significant results $(P<0.05)$ and trends $(0.05 \leq P \leq 0.10)$ from an ANOVA analysis were followed up by post-hoc two-sample T-tests.

\section{Image Acquisition and Preprocessing}

A description of the image acquisition can be found elsewhere [de Wit et al., 2012]. A detailed description of the preprocessing procedure is presented in the Supporting Information text.

\section{Functional Connectivity Analyses}

Two complementary techniques, namely psychophysiological interaction (PPI) analyses and dynamic causal modeling (DCM), were used to examine functional 
connectivity. Regions of interest selection for the DCM analyses was based on the results of the PPI analysis.

\section{Psycho-physiological interaction analysis}

PPI is an analysis technique that is used to examine how functional connectivity between seed regions and the rest of the brain is altered by psychological variables (i.e., task conditions) [Friston et al., 1997]. Ten regions-ofinterest (ROIs) were used for the PPI analysis. As we were primarily interested in connectivity in the response inhibition network, we used ROIs based on the peak voxels from the main task effect [contrast: successful stop $>$ successful go] as previously described [de Wit et al., 2012]. Using Marsbar (http://marsbar.sourceforge.net) we created ROIs by drawing $10 \mathrm{~mm}$ radius spheres around the following peak voxels Montreal Neurological Institute (MNI) coordinates and only including voxels that were inside the main group activation brain mask: inferior frontal gyri (BA 47/ 45/13; right: $x / y / z=33 / 23 /-11,91$ voxels; left $x / y / z$ : 33/ $23 /-8,117$ voxels), inferior parietal cortex (BA 40; right: $x$ / $y / z=42 /-55 / 43,150$ voxels; left: $x / y / z:=-51 /-55 / 43,81$ voxels), pre-SMA (BA 6; right: $x / y / z=9 / 17 / 67$, 80 voxels; left: $x / y / z=-15 / 14 / 67,85$ voxels), and anterior cingulate cortex $(x / y / z=0 / 20 / 34,153$ voxels). We additionally drew a 5-mm sphere around the peak voxel of the subthalamic nucleus $(x / y / z=3 /-15 /-2,17$ voxels). To additionally assess connectivity between the response inhibition network and the limbic system, amygdala ROIs were added (right amygdala [73 voxels]; left amygdala [65 voxels]; masks from the wfu_pickatlas [Maldjian et al., 2003, 2004]).

Imaging data were analyzed in the context of the general linear model. The onsets of successful go (SG) trials and successful stop (SS) trials were modeled with delta functions convolved with a canonical hemodynamic response function. Six regressors were included to correct for movement, and a high-pass filter (128 s cutoff period) was used to remove low-frequency noise.

To create the physiological variable, the time series of the first eigenvariate of the BOLD signal, extracted from our seed regions, was temporally filtered, mean corrected and deconvolved. The PPI interaction variable was then computed as the cross-product of the physiological variable and the psychological variable, that is, the response inhibition contrast (successful stop $>$ successful go). The interaction term was then entered into a regression model, with the psychological and physiological variables included as covariates of no interest in order to remove main effects of task. Using $\mathrm{t}$-contrasts, we assessed the effect of the PPI interaction at the subject-level, [+1] meaning positive coupling with the seed region, $[-1]$ meaning negative coupling with the seed region. Resulting firstlevel contrast images (one per region of interest) were brought into second-level group analyses to assess withingroup and between-group effects on functional connectivity during response inhibition.
Main effects of task were analyzed in one-sample T-tests per group. Effects of group were assessed using a one-way ANOVA and post hoc two-sample T-tests. All analyses included SSRT and gender as covariates of no interest. In addition, SSRT was entered into a regression analysis per group to examine whether the connectivity was associated with task performance.

While PPI examines the connectivity between a seed region and the rest of the brain, given our strong hypotheses within the network we confine our reported results to the connectivity between our regions of interest amongst each other and between our regions of interest and the amygdala. To correct for multiple testing in our PPI analyses, we calculated a new alpha value using the SISA Bonferroni tool (http://www.quantitativeskills.com/sisa/ calculations/bonhlp.htm). To do this, we extracted the beta regressors from our ROIs in the first-level SS > SG contrast, calculated the mean correlation between ROIs $(r=0.47)$ and then used this value and a previous alpha value of $P=0.001$ to calculate a new, corrected alpha value. For the PPI analysis, results survive correction for multiple testing if $P \leq 0.0001723$.

\section{Dynamic causal modeling analysis}

To study the direction of the altered IFG-amygdala connectivity we observed in the PPI analysis, we performed a dynamic causal modeling analysis [Friston et al., 2003]. A brief description of the technique is supplied in the Supporting Information. We included four regions of interest in our DCM models, based on the results of our PPI analyses: the left inferior frontal gyrus (LIFG), right inferior frontal gyrus (RIFG), left amygdala and right amygdala. The notion of direct connections between the IFG and the amygdala is supported by anatomical studies in rhesus monkeys [Amaral and Price, 1984]. Furthermore, a previous effective connectivity study reported bidirectional information flow between these regions during performance of a cognitive task [Curcić-Blake et al., 2012].

For this DCM analysis, fifteen different models were created by varying the intrinsic connections between the IFG and the amygdala. Connections between these regions were either top-down, bottom-up, or both (i.e., bidirectional). The parallel connections between the same regions across hemispheres were assumed to be bidirectional in every model. All connections had modulatory effects of both successful stop and successful go. As it was unclear from previous research whether input was first received by the amygdala or the inferior frontal gyrus or by both regions simultaneously, we created a model space of models with all the possible inputs (to the IFG, to the amygdala or to both). Therefore, in total 45 different models were specified and estimated.

Similar to PPI, DCM uses a volume of interest approach (see for detailed description of the volume of interest extraction procedure the Supporting Information). For DCM analysis, all subjects must show activation in all 
regions of interest [Stephan et al., 2010]. As activation of the left or right amygdala was absent during SS or SG for some subjects at a lenient threshold, not all subjects could be included in the DCM analysis. For our DCM analysis we used imaging data from 22 patients with obsessivecompulsive disorder, 12 unaffected siblings and 20 healthy controls (for demographic and clinical description of these subgroups, see Supporting Information Table S2). Groups were still matched for demographic measures and performance, siblings included in the DCM analysis were significantly younger (mean age 33.2; standard deviation 10.87) than siblings not included in the DCM analysis (mean age 50.54; standard deviation 11.15) $(t[15]=2.975$, $P=0.009$, see Supporting Information Table S3).

A random effects Bayesian model selection (BMS) procedure [Stephan et al., 2009] was used to find the model with the highest exceedance probability, the probability that one model is more likely than other models in each subject group. Bayesian model averaging (BMA) was then used to estimate the strength of specific connections between regions, depicting the representative strength of connection for the whole group. BMA was performed for each group separately and differences in connection strength between our three groups were examined using a previously described bootstrapping method [Curčić-Blake et al., 2012] (see Supporting Information for a description of this method). BMA was also calculated separately for each subject, in order to examine correlations between mean connection strengths and SSRT. As the number of comparisons is considerably smaller for the DCM analyses and these involve aggregated scores rather than voxelbased comparison, the probability of type- 1 errors is markedly reduced. To also reduce the risk for type-2 errors, we used no correction for multiple comparisons in the DCM analyses.

\section{RESULTS}

\section{Task Performance}

As reported previously [de Wit et al., 2012], SSRT was significantly increased in patients compared to controls $(t=2.07, P=0.04)$, whereas siblings did not differ in SSRT from both patients and controls.

\section{Psycho-Physiological Interaction Analyses}

\section{Main effects of task}

During response inhibition, negative coupling between all seed regions was observed in the three groups (data not shown).

\section{Group differences (ANOVA)}

Inhibition-related connectivity group differences were found between the left IFG and small clusters in the bilateral amygdala (see Table I and Fig. 1). Follow-up post-hoc tests showed increased negative coupling between the left inferior frontal gyrus and the right amygdala in OCD patients compared to controls. When results were examined at a more lenient $P$-value (without controlling for multiple comparisons; $P<0.001$ ) clusters were larger and the negative coupling between the left inferior frontal gyrus and amygdala was stronger in both OCD patients and siblings compared to controls (see Supporting Information Table S1). The abovementioned results were not related to SSRT.

\section{Post hoc regression with pre-SMA activation}

To assess how our main PPI results of altered IFGamygdala coupling in OCD and siblings related to our previous finding of hyperactivation of the left-pre-SMA in these groups during response inhibition, we performed a post hoc regression analysis. We extracted the beta estimate from the left pre-SMA region of interest for every individual and used this pre-SMA beta estimate as a regressor in our PPI analysis of the left IFG to examine if there was an interaction between IFG-amygdala connectivity and left pre-SMA activity. Results show that in patients with obsessive-compulsive disorder connectivity between the left IFG and amygdala correlated negatively with taskrelated activity of the left pre-SMA activity $(r=-0.456$, $P=0.003)$, indicating that increased task-related activation of the pre-SMA was accompanied by increased negative

TABLE I. PPI analysis-group differences in functional connectivity during inhibition

\begin{tabular}{|c|c|c|c|c|c|c|c|c|c|c|c|c|c|c|}
\hline \multirow[b]{2}{*}{ Seed } & \multirow[b]{2}{*}{ Region (BA) } & \multicolumn{3}{|c|}{ MNI coordinates } & \multicolumn{3}{|c|}{$\begin{array}{l}\text { Main effect of } \\
\text { group }^{\text {a }}\end{array}$} & \multicolumn{7}{|c|}{ Between group comparisons ${ }^{b}$} \\
\hline & & $X$ & Y & Z & $K_{\mathrm{e}}$ & Z & $P_{\text {unc }}$ & & $X$ & $Y$ & Z & $K_{\mathrm{e}}$ & Z & $P_{\text {unc }}$ \\
\hline L. IFG & R. Amygdala & 30 & -7 & -17 & 2 & 3.80 & 0.000 & $\mathrm{OCD}>\mathrm{HC}$ & 27 & -7 & -17 & 4 & 4.01 & 0.000 \\
\hline L. IFG & L. Amygdala & -30 & -7 & -14 & 1 & 3.59 & 0.000 & & & & & & & \\
\hline
\end{tabular}

BA, Brodmann area; $K_{\mathrm{e}}$, cluster extent; $Z, Z$-score; $P_{\text {unc }}$ uncorrected $P$-value; HC: healthy controls; L., left; R., right; IFG, inferior frontal gyrus.

${ }^{\text {aThree group ANOVA. }}$

${ }^{\mathrm{b}}$ Only significant results are reported $(P \leq 0.0001723)$. Post hoc two-sample $T$-test. 
a

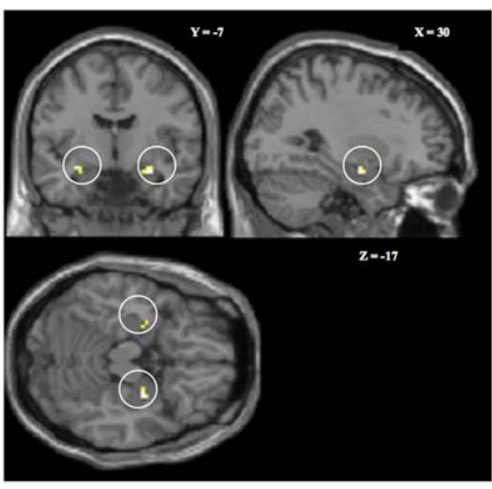

b

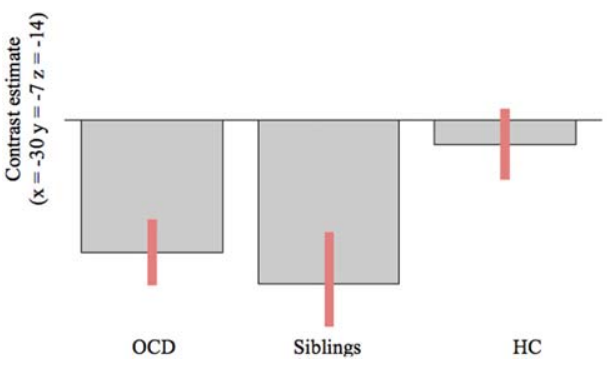

C

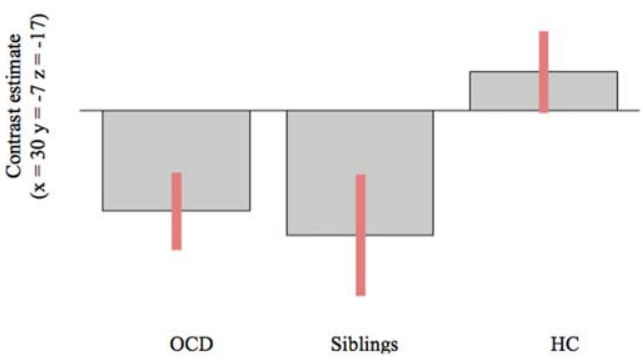

Figure I.

(a) Altered IFG-amygdala functional connectivity in OCD and unaffected siblings. Results of PPI analysis, Regions that show group differences in functional connectivity with the left inferior frontal gyrus during successful response inhibition (successful stop $>$ successful go), including the left and right amygdala $(P=0.00$ I, uncorrected $)$. (b) Contrast estimates at $(x=-30$, $y=-7, z=-14)$. Negative coupling between the left inferior frontal gyrus and the left amygdala is seen in OCD patients and siblings, but is absent in healthy controls. (c) Contrast estimates at $(x=30, y=-7, z=-17)$. Negative coupling between the left inferior frontal gyrus and the right amygdala is seen in OCD patients and siblings, but is absent in healthy controls. coupling between the IFG and the amygdala (see Fig. 2). The relationship between IFG-amygdala connectivity and pre-SMA activity was also seen in siblings $(r=-0.650$, $P=0.005)$, but was after removal of an outlier only seen at trend-level $(r=-0.459, P=0.074)$.

\section{Dynamic Causal Modeling}

Results of the BMS did not reveal a winning model in the healthy control group, as the exceedance probabilities for multiple models that included bottom-up connections were equally high (see Supporting Information Table S3 and Fig. S1), indicating multiple winning models in this group. In patients with obsessive-compulsive disorder, the model with the highest exceedance probability was a model that had a top-down connection from the left IFG to the left amygdala (see Supporting Information Fig. S1). In siblings, the winning model included bidirectional connections between the IFG and amygdala in both hemispheres (see Supporting Information Fig S1) and had an exceedance probability of $28 \%$. However, the winning model for patients (i.e., top-down connection in the left hemisphere) was only slightly worse in siblings, with an exceedance probability of $21 \%$. In all three participant groups, models with the highest exceedance probability received input in the bilateral inferior frontal gyrus (see Supporting Information Fig. S1).

To investigate differences between groups in connection strengths, we performed BMA per group (for results see Table II). The strength of the connection between the left amygdala and left IFG was reduced in siblings compared to patients with obsessive-compulsive disorder (in $96 \%$ of samples) and healthy controls (in 95\% of all samples). The mean connection strength was positive in patients with obsessive-compulsive disorder and controls, but was negative in siblings. There was no difference in connection strength between subject groups during the task conditions (successful stop and successful go).

BMA was also calculated for each subject separately and compared between groups using a non-parametric Kruskal-Wallis test. No significant group differences were 
observed. The connectivity strength from the left amygdala to the left IFG, which differed between groups in the BMA per group analysis, showed a negative correlation with SSRT in healthy controls $(r=-0.492, P=0.03)$.

\section{DISCUSSION}

The present study is the first to examine the functional integrity of a response inhibition network in patients with obsessive-compulsive disorder and their unaffected siblings in comparison with healthy controls. Results of our PPI analysis show abnormal negative coupling between the left IFG and the bilateral amygdala in patients and at a more lenient threshold also in siblings, and additionally between the right inferior frontal gyrus and the right amygdala in patients only. These findings suggests that altered left IFG-amygdala connectivity interferes with taskrelated activation of the inhibition network. Follow-up DCM BMS analysis showed different winning models in each subject group, indicative of altered IFG-amygdala connectivity in patients and siblings during both successful stop and successful go trials.

In our previous study, we examined brain activation during performance of a response inhibition task in the same participants [de Wit et al., 2012] and observed compensatory hyperactivity of the left pre-SMA during response inhibition in patients with obsessive-compulsive disorder and their siblings. In the current study, increased negative connectivity between the IFG and amygdala was associated with increased activation of the left pre-SMA in patients and at trend-level in siblings. We hypothesize that altered connectivity between the IFG and amygdala in patients and siblings leads to inefficient processing in frontal-striatal circuits, resulting in compensatory taskrelated hyperactivation of the pre-SMA. This is in accordance with neurobiological models of obsessive-compulsive disorder, which suggest interference between frontalstriatal circuits and the amygdala [Mataix-Cols and van den Heuvel, 2006; van den Heuvel et al., in press].

Despite finding different winning models in each group in the BMS analysis, we were unable to find group differences in the modulatory effect of SS and SG on the strength of IFG-amygdala connections. We did observe decreased general connection strength in siblings between the left amygdala and the left IFG. This seems to suggest a general change in IFG-amygdala connectivity in this group. However, as the DCM analysis was performed on a subset of participants, we may have had insufficient power to show task-condition modulatory effects.

Our main finding thus is altered IFG-amygdala connectivity during response inhibition in patients with obsessive-compulsive disorder and siblings. The inferior frontal gyrus is crucial for response inhibition [Chambers et al., 2006] and is involved in both the detection of the stop signal and generation of inhibitory output [Chikazoe et al., 2009; Levy and Wagner, 2011]. Although many studies
A.
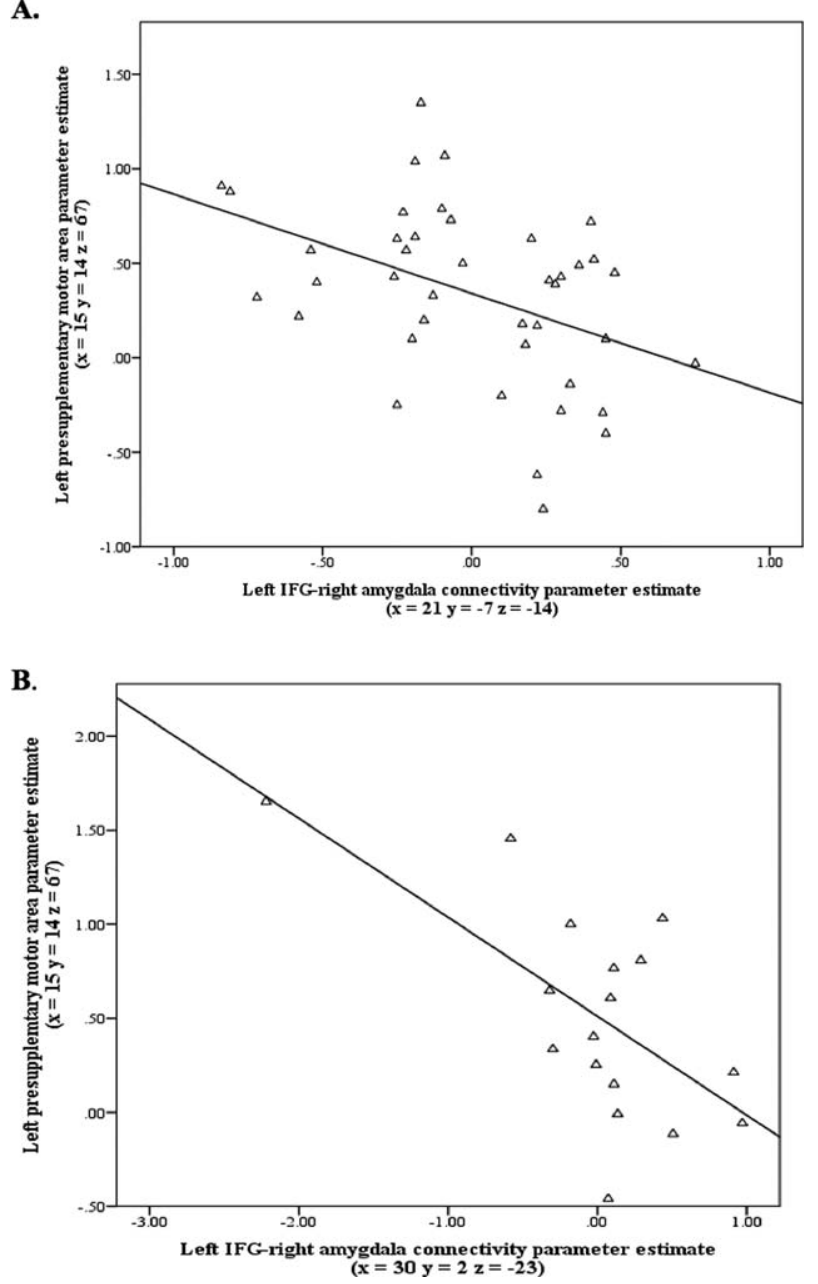

Figure 2.

Relationship between observed endophenotype of altered IFGamygdala functional coupling in OCD patients and siblings and previously described task-related pre-SMA hyperactivity in these groups [de Wit et al., 2012]. Correlations between activation of the left pre-SMA and the connectivity between the left inferior frontal gyrus and right amygdala in patients (A) and siblings (B). The relationship in siblings was only seen at a trend-level after removal of an outlier $(P=0.074)$.

implicate the right IFG in inhibition, the left IFG seems also involved [Duann et al., 2009; Swick et al., 2008]. Both the right IFG [Page et al., 2009; de Wit et al., 2012; Woolley et al., 2008] and left IFG [Maltby et al., 2005; Page et al., 2009] have been found to be hypoactive during response inhibition in patients with obsessive-compulsive disorder, which may reflect decreased attention allocation to the stop signal or an impairment in generating the neural stop signal.

Many studies have emphasized the role of frontalstriatal connections in obsessive-compulsive disorder, although studies also showed involvement of the limbic 
- Connectivity during Inhibition in OCD •

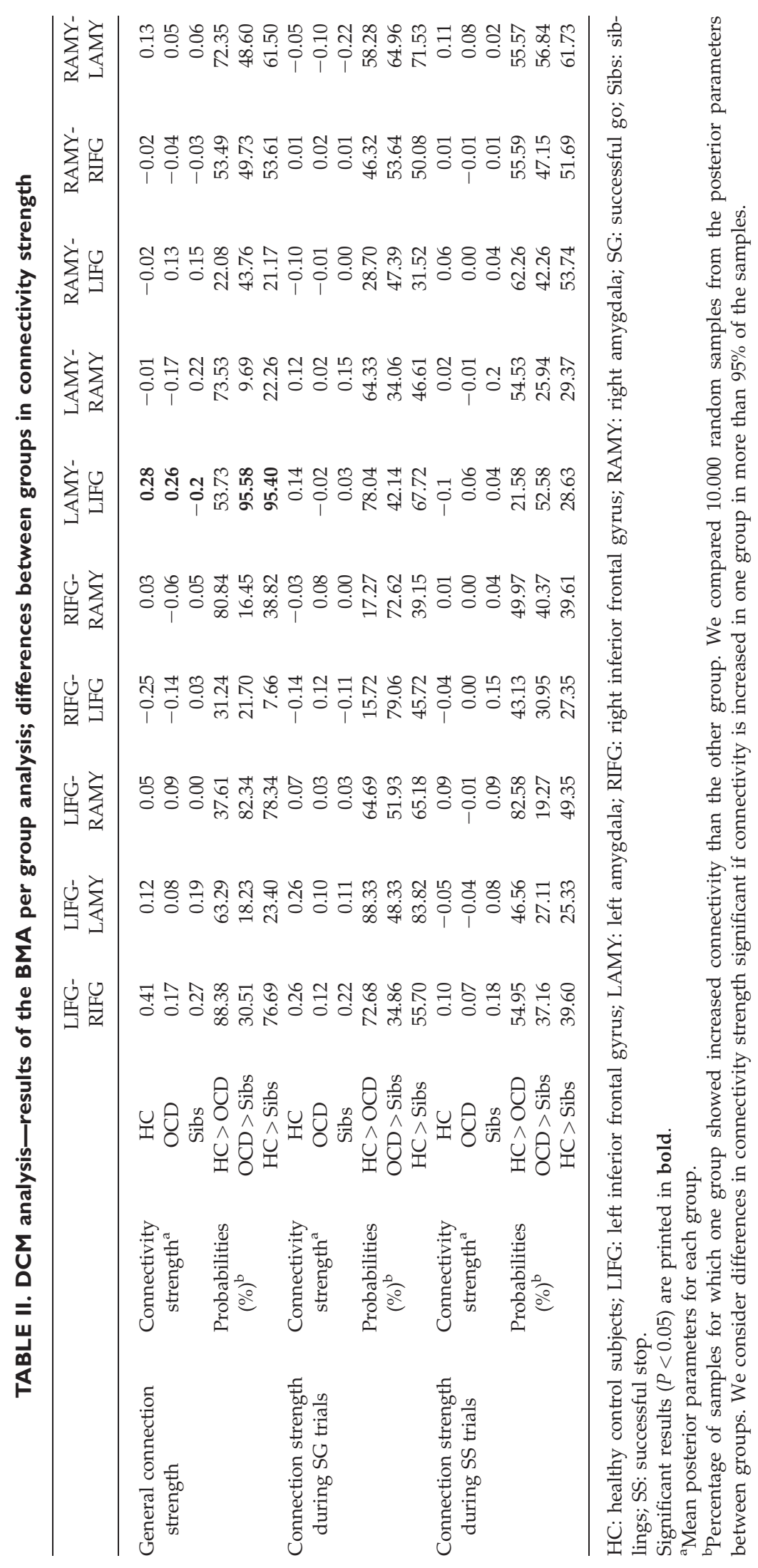


system, including the amygdala [Admon et al., 2012; Cardoner et al., 2011; Milad and Rauch, 2012] in the disorder. Studies in healthy samples showed that the amygdala plays a role in motor inhibition in response to threat [Sagaspe et al., 2011] and in the detection of behaviorally relevant or salient stimuli in other cognitive tasks [Kiehl et al., 2005; Ousdal et al., 2008]. Altered connectivity of the amygdala during response inhibition performance in patients with obsessive-compulsive disorder has never been reported before. Some studies, however, have observed structural abnormalities of the amygdala in obsessive-compulsive disorder [Kwon et al., 2003; Szeszko et al., 1999] and increased amygdala activation during decision making and processing of emotional faces or disease-relevant emotional stimuli [Cardoner et al., 2011; Stern et al., 2013; van den Heuvel et al., 2004].

Results of the DCM analysis indicated that the amygdala had a bottom-up effect on the IFG during task performance in healthy controls and siblings. This is in line with previous suggestions that detection of salient or goalrelevant stimuli (in this case the stop signal) or vigilance in response to uncertain events (the presentation of the stop signal) may be mediated by the amygdala and that the amygdala may send modulatory signals to the cortex affecting attention allocation or causing behavioral adaptation [Davis and Whalen, 2001; Schaefer et al., 2006; Schaefer and Gray, 2007]. Thus, a bottom-up effect of the amygdala on the prefrontal cortex may enhance attention to the stop signal and speed during inhibitory performance. This putative performance-enhancing effect of the amygdala on the IFG appears to be absent in patients with obsessive-compulsive disorder, as task-related processing occurred top-down in this group. At first glance, this may appear counterintuitive, as many models of obsessivecompulsive disorder speculate that limbic regions exert increased influence over prefrontal areas in obsessivecompulsive disorder [Mataix-Cols and van den Heuvel, 2006]. We hypothesize that the stop signal may be more salient in patients with obsessive-compulsive disorder and that increased activation of the amygdala may therefore need to be suppressed by the IFG in the patient group. Previous studies strengthen this notion by showing altered brain activation in response to salient stimuli using oddball tasks in patients with obsessive-compulsive disorder [Kim et al., 2003; Rubia et al., 2011]. As we did not find significant correlations between SSRT and IFG-amygdala connectivity in patients or siblings, though it was related to pre-SMA hyperactivity (which was correlated with SSRT in our previous study) in patients, it remains unclear how our results relate directly to the response inhibition impairment in obsessive-compulsive disorder.

Abnormal IFG-amygdala connectivity during response inhibition was also seen in unaffected siblings of patients with obsessive-compulsive disorder at a lenient statistical threshold (see Supporting Information), which suggests that it is related to the genetic susceptibility for obsessive- compulsive disorder. Previous imaging genetics studies in obsessive-compulsive disorder showed that the dopamine receptor D2 polymorphism is associated with altered functional connectivity between the amygdala and the dorsolateral prefrontal cortex during emotional processing [Blasi et al., 2009]. Furthermore, the met allele of the COMT gene has been associated with increased extracellular dopamine levels [Lachman et al., 1996] and increased connectivity between the amygdala and orbitofrontal cortex [Rasch et al., 2010] and between the amygdala and IFG [Drabant et al., 2006] during processing of emotional stimuli. Both abovementioned genes have been associated with obsessive-compulsive disorder in several studies [Denys et al., 2006; Karayiorgou et al., 1997; Schindler et al., 2000]. Additionally, polymorphisms of the serotonin transporter gene affect fronto-limbic connectivity [Long et al., 2013; Surguladze et al., 2012] and have been associated with obsessive-compulsive disorder [Lin, 2007]. Future research is needed to assess whether the altered IFG-amygdala connectivity during response inhibition we observed in patients and siblings is related to these dopaminergic and serotonergic genes.

Strengths of the present study are the inclusion of medication-free subjects and use of two complementary techniques to assess functional connectivity (PPI and DCM). A limitation of our PPI analysis is the use of large regions of interest $(10 \mathrm{~mm})$ that were similar across all subjects, instead of the use of smaller subject-specific regions of interest, like those needed for [Stephan et al., 2010] and used in the DCM analysis. We chose to use larger regions of interest in the PPI analysis to relate current findings to our previous results obtained with these exact regions of interest [de Wit et al., 2012]. Additionally, Passamonti et al. [2008] found comparable PPI results using both large subject-specific regions of interest and a region of interest that was similar across all subjects. Because of the use of subject-specific regions of interest in DCM, not all subjects in the PPI analysis could be included in our DCM analysis, which may warrant replication in a larger sample. However, in our DCM analysis groups were still matched for demographic and clinical variables. Additionally, in a post hoc analysis (data not shown), we were able to replicate the original PPI effects in the smaller sample of subjects included in the DCM analysis, in the left hemisphere (yet at a more lenient threshold).

\section{CONCLUSION}

To conclude, our findings suggest that altered IFGamygdala connectivity in obsessive-compulsive disorder, and to a lesser extent in their unaffected siblings, interferes with the task-related efficiency of the response inhibition network resulting in compensatory pre-SMA hyperactivation. Our results add a relevant piece of knowledge in the context of the discussion on the role of anxiety and limbic interference in obsessive-compulsive disorder. 


\section{ACKNOWLEDGMENTS}

The authors would like to thank C. Vriend, MSc, and N.J.H.M. Gerrits, MSc, for their input and suggestions during the data-analysis of this study. The authors would also like to thank E.M. Veltman, MD, V. van der Borden, MSc, and S.J.C. Verfaillie, MSc, for their assistance during data acquisition. Author contributions: S.J.d.W., Y.D.v.d.W., D.J.V. and O.A.v.d.H. were involved in design of the study; S.J.d.W., F.E.d.V., Y.D.v.d.W. and O.A.v.d.H. were involved in data acquisition; S.J.d.W., O.A.v.d.H. and L.S.v.V. performed the data analyses; all authors contributed to the interpretation of the data, revised earlier drafts of this article and have approved the final version of the manuscript and its submission.

\section{REFERENCES}

Admon R, Bleich-Cohen M, Weizmant R, Poyurovsky M, Faragian S, Hendler T (2012): Functional and structural neural indices of risk aversion in obsessive-compulsive disorder (OCD). Psychiatry Res 203:207-213.

Amaral DG, Price JL (1984): Amygdalo-cortical projections in the monkey (Macaca fascicularis). J Comp Neurol 230:465-496..

Aron AR (2011): From reactive to proactive and selective control: Developing a richer model for stopping inappropriate responses. Biol Psychiatry 69:e55-e68.

Aron AR, Poldrack RA (2006): Cortical and subcortical contributions to Stop signal response inhibition: Role of the subthalamic nucleus. J Neurosci 26:2424-2433.

Bannon S, Gonsalvez CJ, Croft RJ, Boyce PM (2002): Response inhibition deficits in obsessive-compulsive disorder. Psychiatry Res 110:165-174.

Blasi G, Lo Bianco L, Taurisano P, Gelao B, Romano R, Fazio L, Papazacharias A, Di Giorgio A, Caforio G, Rampino A, Masellis R, Papp A, Ursini G, Sinibaldi L, Popolizio T, Sadee W, Bertolino A (2009): Functional variation of the dopamine D2 receptor gene is associated with emotional control as well as brain activity and connectivity during emotion processing in humans. J Neurosci 29:14812-14819.

Cardoner N, Harrison BJ, Pujol J, Soriano-Mas C, HernándezRibas R, López-Solá M, Real E, Deus J, Ortiz H, Alonso P, Menchón JM (2011): Enhanced brain responsiveness during active emotional face processing in obsessive compulsive disorder. World J Biol Psychiatry 12:349-363.

Chamberlain SR, Fineberg NA, Blackwell AD, Robbins TW, Sahakian BJ (2006): Motor inhibition and cognitive flexibility in obsessive-compulsive disorder and trichotillomania. Am J Psychiatry 163:1282-1284.

Chamberlain SR, Fineberg NA, Menzies LA, Blackwell AD, Bullmore ET, Robbins TW, Sahakian BJ (2007): Impaired cognitive flexibility and motor inhibition in unaffected first-degree relatives of patients with obsessive-compulsive disorder. Am J Psychiatry 164:335-338.

Chambers CD, Bellgrove MA, Stokes MG, Henderson TR, Garavan H, Robertson IH, Morris AP, Mattingley JB (2006): Executive "brake failure" following deactivation of human frontal lobe. J Cogn Neurosci 18:444-455.

Chambers CD, Garavan H, Bellgrove MA (2009): Insights into the neural basis of response inhibition from cognitive and clinical neuroscience. Neurosci Biobehav Rev 33:631-646.
Chen C-Y, Muggleton NG, Tzeng OJL, Hung DL, Juan C-H (2009): Control of prepotent responses by the superior medial frontal cortex. Neuroimage 44:537-545.

Chikazoe J, Jimura K, Asari T, Yamashita K, Morimoto H, Hirose S, Miyashita Y, Konishi S (2009): Functional dissociation in right inferior frontal cortex during performance of go/no-go task. Cereb Cortex 19:146-152.

Curcić-Blake B, Swart M, Aleman A (2012): Bidirectional information flow in frontoamygdalar circuits in humans: A dynamic causal modeling study of emotional associative learning. Cereb Cortex 22:436-445.

Curčić-Blake B, Swart M, Ter Horst GJ, Langers DRM, Kema IP, Aleman A (2012): Variation of the gene coding for DARPP-32 (PPP1R1B) and brain connectivity during associative emotional learning. Neuroimage 59:1540-1550.

Davis M, Whalen PJ (2001): The amygdala: Vigilance and emotion. Mol Psychiatry 6:13-34.

Denys D, Van Nieuwerburgh F, Deforce D, Westenberg H (2006): Association between the dopamine D2 receptor TaqI A2 allele and low activity COMT allele with obsessive-compulsive disorder in males. Eur Neuropsychopharmacol 16:446-450.

Drabant EM, Hariri AR, Meyer-Lindenberg A, Munoz KE, Mattay VS, Kolachana BS, Egan MF, Weinberger DR (2006): Catechol O-methyltransferase val158met genotype and neural mechanisms related to affective arousal and regulation. Arch Gen Psychiatry 63:1396-1406.

Duann J-R, Ide JS, Luo X, Li CR (2009): Functional connectivity delineates distinct roles of the inferior frontal cortex and presupplementary motor area in stop signal inhibition. J Neurosci 29:10171-10179.

Fontenelle LF, Mendlowicz MV, Versiani M (2006): The descriptive epidemiology of obsessive-compulsive disorder. Prog Neuropsychopharmacol Biol Psychiatry 30:327-337.

Friston KJ, Buechel C, Fink GR, Morris J, Rolls E, Dolan RJ (1997): Psychophysiological and modulatory interactions in neuroimaging. Neuroimage 6:218-229.

Friston KJ, Harrison L, Penny W (2003): Dynamic causal modelling. Neuroimage 19:1273-1302.

Van den Heuvel OA, Mataix-Cols D, Zwitser G, Cath DC, van der Werf YD, Groenewegen HJ, van Balkom AJLM, Veltman DJ (2011): Common limbic and frontal-striatal disturbances in patients with obsessive compulsive disorder, panic disorder and hypochondriasis. Psychol Med 41:2399-2410.

Van den Heuvel OA, van Wingen G, Soriano-Mas C, Alonso P, Chamberlain SR, Nakamae T, Denys D, Goudraiaan AE, Veltman DJ: Brain circuitry of compulsivity. Eur Neuropsychopharmacol (in press).

Van den Heuvel OA, Veltman DJ, Groenewegen HJ, Dolan RJ, Cath DC, Boellaard R, Mesina CT, van Balkom AJLM, van Oppen P, Witter MP, Lammertsma AA van Dyck R (2004): Amygdala activity in obsessive-compulsive disorder with contamination fear: A study with oxygen-15 water positron emission tomography. Psychiatry Res 132:225-37.

Van den Heuvel OA, Veltman DJ, Groenewegen HJ, Witter MP, Merkelbach J, Cath DC, van Balkom AJLM, van Oppen P van Dyck R (2005): Disorder-specific neuroanatomical correlates of attentional bias in obsessive-compulsive disorder, panic disorder, and hypochondriasis. Arch Gen Psychiatry 62:922-33.

Kang D-H, Jang JH, Han JY, Kim J-H, Jung WH, Choi J-S, Choi CH, Kwon JS (2013): Neural correlates of altered response inhibition and dysfunctional connectivity at rest in obsessivecompulsive disorder. Prog Neuropsychopharmacol Biol Psychiatry 40:340-346. 
Karayiorgou M, Altemus M, Galke BL, Goldman D, Murphy DL, Ott J, Gogos JA (1997): Genotype determining low catechol-Omethyltransferase activity as a risk factor for obsessivecompulsive disorder. Proc Natl Acad Sci USA 94:4572-4575.

Kiehl KA, Stevens MC, Laurens KR, Pearlson G, Calhoun VD, Liddle PF (2005): An adaptive reflexive processing model of neurocognitive function: Supporting evidence from a large scale $(n=100)$ fMRI study of an auditory oddball task. Neuroimage 25:899-915.

Kim M-S, Kang S-S, Youn T, Kang D-H, Kim J-J, Kwon JS (2003): Neuropsychological correlates of P300 abnormalities in patients with schizophrenia and obsessive-compulsive disorder. Psychiatry Res 123:109-123.

Kwon JS, Shin Y-W, Kim C-W, Kim YI, Youn T, Han MH, Chang K-H, Kim J-J (2003): Similarity and disparity of obsessivecompulsive disorder and schizophrenia in MR volumetric abnormalities of the hippocampus-amygdala complex. J Neurol Neurosurg Psychiatry 74:962-964.

Lachman HM, Papolos DF, Saito T, Yu YM, Szumlanski CL, Weinshilboum RM (1996): Human catechol-O-methyltransferase pharmacogenetics: Description of a functional polymorphism and its potential application to neuropsychiatric disorders. Pharmacogenetics 6:243-250.

Levy BJ, Wagner AD (2011): Cognitive control and right ventrolateral prefrontal cortex: Reflexive reorienting, motor inhibition, and action updating. Ann N Y Acad Sci 1224:40-62.

Lin P-Y (2007): Meta-analysis of the association of serotonin transporter gene polymorphism with obsessive-compulsive disorder. Prog Neuropsychopharmacol Biol Psychiatry 31:683-689.

Logan GD (1994): On the ability to inhibit thought and action: A users' guide to the stop signal paradigm. In: Dagenbach D, Carr $\mathrm{TH}$, editors. Inhibitory processes in attention, memory and language. San Diego: Academic Press. pp 189-239.

Logan GD, Cowan WB (1984): On the ability to inhibit thought and action: A theory of an act of control. Psychological Review 91:295-327.

Long H, Liu B, Hou B, Wang C, Li J, Qin W, Wang D, Zhou Y, Kendrick KM, Yu C, Jiang T (2013): The long rather than the short allele of 5-HTTLPR predisposes Han Chinese to anxiety and reduced connectivity between prefrontal cortex and amygdala. Neurosci Bull 29:4-15.

Maldjian JA, Laurienti PJ, Burdette JH (2004): Precentral gyrus discrepancy in electronic versions of the Talairach atlas. Neuroimage 21:450-455.

Maldjian JA, Laurienti PJ, Kraft RA, Burdette JH (2003): An automated method for neuroanatomic and cytoarchitectonic atlasbased interrogation of fMRI data sets. Neuroimage 19:1233-1239.

Maltby N, Tolin DF, Worhunsky P, O'Keefe TM, Kiehl KA (2005): Dysfunctional action monitoring hyperactivates frontal-striatal circuits in obsessive-compulsive disorder: An event-related fMRI study. Neuroimage 24:495-503.

Mataix-Cols D, van den Heuvel OA (2006): Common and distinct neural correlates of obsessive-compulsive and related disorders. Psychiatr Clin North Am 29:391-410, viii.

Milad MR, Rauch SL (2012): Obsessive-compulsive disorder: Beyond segregated cortico-striatal pathways. Trends Cogn Sci 16:43-51.

Ousdal OT, Jensen J, Server A, Hariri AR, Nakstad PH, Andreassen OA (2008): The human amygdala is involved in general behavioral relevance detection: Evidence from an event-related functional magnetic resonance imaging Go-NoGo task. Neuroscience 156:450-455.

Page LA, Rubia K, Deeley Q, Daly E, Toal F, Mataix-Cols D, Giampietro V, Schmitz N, Murphy DGM (2009): A functional magnetic resonance imaging study of inhibitory control in obsessive-compulsive disorder. Psychiatry Res 174:202-209.

Passamonti L, Rowe JB, Ewbank M, Hampshire A, Keane J, Calder AJ (2008): Connectivity from the ventral anterior cingulate to the amygdala is modulated by appetitive motivation in response to facial signals of aggression. Neuroimage 43:562570.

Rasch B, Spalek K, Buholzer S, Luechinger R, Boesiger P, de Quervain DJ-F, Papassotiropoulos A (2010): Aversive stimuli lead to differential amygdala activation and connectivity patterns depending on catechol-O-methyltransferase Val158Met genotype. Neuroimage 52:1712-1719.

Rubia K, Cubillo A, Woolley J, Brammer MJ, Smith A (2011): Disorder-specific dysfunctions in patients with attention-deficit/ hyperactivity disorder compared to patients with obsessivecompulsive disorder during interference inhibition and attention allocation. Hum Brain Mapp 32:601-611.

Ruscio AM, Stein DJ, Chiu WT, Kessler RC (2010): The epidemiology of obsessive-compulsive disorder in the National Comorbidity Survey Replication. Mol Psychiatry 15:53-63.

Sagaspe P, Schwartz S, Vuilleumier P (2011): Fear and stop: A role for the amygdala in motor inhibition by emotional signals. Neuroimage 55:1825-1835.

Schaefer A, Braver TS, Reynolds JR, Burgess GC, Yarkoni T, Gray JR (2006): Individual differences in amygdala activity predict response speed during working memory. J Neurosci 26:1012010128.

Schaefer A, Gray JR (2007): A role for the human amygdala in higher cognition. Rev Neurosci 18:355-363.

Schindler KM, Richter MA, Kennedy JL, Pato MT, Pato CN (2000): Association between homozygosity at the COMT gene locus and obsessive compulsive disorder. Am J Med Genet 96:721-724.

Schlösser RGM, Wagner G, Schachtzabel C, Peikert G, Koch K, Reichenbach JR, Sauer H (2010): Fronto-cingulate effective connectivity in obsessive compulsive disorder: A study with fMRI and dynamic causal modeling. Hum Brain Mapp 31:1834-1850.

Stephan KE, Penny WD, Moran RJ, den Ouden HEM, Daunizeau J, Friston KJ (2010): Ten simple rules for dynamic causal modeling. Neuroimage 49:3099-3109.

Stephan KE, Penny WD, Daunizeau J, Moran RJ, Friston KJ (2009): Bayesian model selection for group studies. Neuroimage 46: 1004-1017.

Stern ER, Welsh RC, Gonzalez R, Fitzgerald KD, Abelson JL, Taylor SF (2013): Subjective uncertainty and limbic hyperactivation in obsessive-compulsive disorder. Hum Brain Mapp 34: 1956-1970.

Surguladze SA, Radua J, El-Hage W, Gohier B, Sato JR, Kronhaus DM, Proitsi P, Powell J, Phillips ML (2012): Interaction of catechol O-methyltransferase and serotonin transporter genes modulates effective connectivity in a facial emotion-processing circuitry. Transl Psychiatry 2:e70.

Swick D, Ashley V, Turken AU (2008): Left inferior frontal gyrus is critical for response inhibition. BMC Neurosci 9:102.

Szeszko PR, Robinson D, Alvir JM, Bilder RM, Lencz T, Ashtari M, Wu H, Bogerts B (1999): Orbital frontal and amygdala volume reductions in obsessive-compulsive disorder. Arch Gen Psychiatry 56:913-919.

De Vries FE, de Wit SJ, Cath DC, van der Werf YD, van der Borden V, van Rossum TB, van Balkom AJLM, van der Wee NJA, Veltman DJ, van den Heuvel OA (2014): Compensatory frontoparietal activity during working memory: An endophenotype of obsessive-compulsive disorder. Biol Psychiatry $878-887$. 
De Wit SJ, Alonso P, Schweren L, Mataix-Cols D, Lochner C, Menchón JM, Stein DJ, Fouche J-P, Soriano-Mas C, Sato JR, Hoexter MQ, Denys D, Nakamae T, Nishida S, Kwon JS, Jang JH, Busatto GF, Cardoner N, Cath DC, Fukui K, Jung WH, Kim SN, Miguel EC, Narumoto J, Phillips ML, Pujol J, Remijnse PL, Sakai Y, Shin NY, Yamada K, Veltman DJ, van den Heuvel OA (2014): Multicenter voxel-based morphometry mega-analysis of structural brain scans in obsessivecompulsive disorder. Am J Psychiatry 171:340-349.
De Wit SJ, de Vries FE, van der Werf YD, Cath DC, Heslenfeld DJ, Veltman EM, van Balkom AJLM, Veltman DJ, van den Heuvel OA (2012): Presupplementary motor area hyperactivity during response inhibition: A candidate endophenotype of obsessive-compulsive disorder. Am J Psychiatry 169:1100-1108. Woolley J, Heyman I, Brammer M, Frampton I, McGuire PK, Rubia K (2008): Brain activation in paediatric obsessive compulsive disorder during tasks of inhibitory control. Br J Psychiatry 192:25-31. 